\title{
2 Geographische Lage und klimatische Verhältnisse des Untersuchungsgebietes
}

Das Siebenstromland (kasach. Жетысу [Žetysu], russ. Семиречье) ist eine historische Landschaft im südöstlichen Kasachstan innerhalb Zentralasiens. Politisch gehört es heute größtenteils zur Republik Kasachstan, seine südlichen und südöstlichen Randgebiete zu Kirgisistan und der chinesischen autonomen Region Xinjang (Karte I).

Der kasachische Eigenname Žetysu besteht aus zwei Wörtern: „žety“ - „Sieben“ und „su“ - „Wasser, Fluss, Strom“. Davon wurden auch die Bezeichnungen für dieses Gebiet in den anderen Sprachen abgeleitet. Nach E. M. Pospelov bezeichnet „sieben“ keine reale Zahl von Gewässern, sondern bedeutet in übertragenem Sinne „viel“ und benennt in erster Linie ein wasserreiches Gebiet im Unterschied zum wasserarmen, relativ trockenen inneren bzw. zentralen Kasachstan (Pospelov I998, 379). Nichtsdestotrotz lassen sich für das Siebenstromland sieben Hauptflüsse feststellen: Ili, Karatal, Sarkand, Bien, Aksu, Lepsa und Baskan (Karte 2). Sie durchfließen die Ebene, die von den Bergketten des nördlichen Tien-Shan, Transili-Alatau und Dsungarischen Alatau im Süden nach Norden abfällt, bis sie in die nördlich gelegenen Seen Balchasch, Sassykol und Alakol münden (Gass (im Druck); Gass 20ırb, 2io). Als östliche Grenze gelten die östlichen Ausläufer des Dsungarischen Alatau und das Alakol-Becken. Im Westen wird das Siebenstromland durch die Taukum-Wüste und die Ču-Ili-Berge begrenzt (Abolin I930, 4, Abb. I; Machett u.a. 2006, I93-I94, Fig. I).

Die geographischen Untersuchungen sowie Fragen der Landschaftsklassifikation, Geologie, Geomorphologie, Orographie, Hydrologie und zum Klima im Siebenstromland wurden im Zusammenhang mit der vorliegenden archäologischen Studie von Maik Blättermann (Blättermann 2013) beschrieben und diskutiert. Aus diesem Grund werden solche Fragen hier nicht näher behandelt. Dennoch sollen die „Lebensräume“ des Siebenstromlandes, die von R. I. Abolin (I930, I66-I74) erarbeitet wurden, kurz zur Charakterisierung des Raumes erwähnt werden, weil Blättermanns Arbeit einen anderen Schwerpunkt setzt. Der Geobotaniker R. I. Abolin gliederte das Siebenstromland auf Grund von Klima, Boden, Vegetation und Fauna von Nord nach Süd in fünf sog. „Lebensräume“. Dabei handelte es sich im Einzelnen um:

I. die Zone der Artemisia-Steppe, die aus einem Artemisia-Steppen- und einem Süßgräser-Artemisia-Steppen-Gürtel besteht. Diese Zone liegt auf einer Höhe von $850-950 \mathrm{~m}$ ü. NN und nimmt das Flachland und eine leicht hügelige Landschaft mit seltenen Sandhügeln ein und weist im Norden helleren und im Süden dunkleren Sierozem-Boden auf. Das Klima des nördlich gelegenen ArtemisiaSteppen-Gürtels ist durch hohe Sommertemperaturen und geringen Niederschlag charakterisiert. Hier wächst überwiegend in inselartiger Verbreitung das namengebende Wermutkraut (Artemisia absinthium). Die übrige Oberfläche bleibt kahl. Der Ackerbau ist hier nur in geringem Ausmaß und mit künstlicher Bewässerung möglich. Daher wird dieses Gebiet hauptsächlich zur saisonalen Winterviehweide genutzt.

Der südlich gelegene Süßgräser-Artemisia-Steppen-Gürtel weist ein ebenso trockenes Klima mit hohen Sommertemperaturen auf, allerdings ist der jährliche Niederschlag hier wesentlich höher. Hier wachsen grauer Wermut (Artemisia terrae-albae), Federgras (Stipa) und echter Schaf-Schwinger (Festuca ovina). Mit künstlicher Bewässerung sind Ackerbau und Gartenbau möglich. Es wird Trockenfeldbau betrieben. Das Gebiet wird dennoch als Weidegebiete für Schafe und Kamele genutzt. 
II. die Zone der Süßgräser-Steppe besteht aus einem nördlich gelegenen Artemisia-Süßgräser-Steppen-Gürtel und einem südlich gelegenen Süßgräser-Steppen-Gürtel. Sie befindet sich auf einer Höhe von 850-I500 $\mathrm{m}$ ü. NN und umfasst Flachland und eine hügelige Landschaft, stellenweise auch mit Bergkuppen. Als Bodenart herrscht Kastanozem vor. Das Klima annähernd gemäßigt, mit wesentlich höheren Niederschlägen als in der ersten Zone. Im nördlich gelegenen Artemisia-Süßgräser-Steppen-Gürtel überwiegen die Süßgräser (Poeceae) gegenüber dem grauen Wermut. Der Trockenfeldbau ist in dieser gebirgsfernen Landschaft möglich, mit künstlicher Bewässerung auch alle Formen des Ackerbaus. Der Artemisia-Süßgräser-Steppen-Gürtel bietet exzellente Weidegebiete für Schafe, Ziegen und Pferde.

Der südlicher gelegene Süßgräser-Steppen-Gürtel liegt in den Vorgebirgen. Hier gibt es keinen grauen Wermut mehr, dafür kommen zu den zahlreichen Süßgräsern verschiedene Strauchpflanzen, namentlich Hagebutten (Rosa), Mädesüß (Filipendula) und Sauerkirsche (Prunus cerasus). Der Ackerbau ist hier auch ohne Bewässerung möglich. Der ganze Gürtel eignet sich als Weidegebiet für Schafe, Ziegen, Rinder und Pferde, allerdings nur in den Übergangsjahreszeiten. In den Wintermonaten ist der Schnee für die Tiere zu hoch.

III. die Zone der gemischten Gras-Steppe liegt in der Vorgebirgslandschaft auf einer Höhe von 800-I80o m ü. NN. Hier herrscht ein gemäßigtes Klima mit warmen, niederschlagsreichen Sommern. In diesem Waldsteppengebiet herrscht das Tschernosem als Bodenart vor. Im unteren Bereich, dem sog. Gürtel der gemischten Gras-Steppe, besteht die Vegetation aus verschiedenen Gräsern, unter denen die Süßgräser dominieren - die Quecke (Elymus), die Rispengräser (Poa) und das Steppen-Lieschgras (Phleum phleoides). An den Hängen der Vorgebirge wachsen Hagebutte, Mädesüß, Weißdorn (Crataegus) und Berberitze (Berberis). Der Ackerbau ist möglich, allerdings nicht überall. Die am besten dafür geeigneten Gebiete befinden sich zwischen Almaty und Turgen, im Umland von Issyk, Kegen, Kaskelen, Karakastek und am Ostufer des Issyk-Kul-Sees. Diese Zone ist in den Übergangsjahreszeiten auch gut als Weidegebiet geeignet. In den Wintermonaten liegt hier sehr viel Schnee, so dass keine Weidemöglichkeit besteht.

Im oberen Bereich dieser Zone, dem sogenannten Laubwald-Gürtel, besteht die Vegetation vor allem aus Apfelbaum (Malus), Weißdorn, Aprikose (Prunus armeniaca), Espe (Populus tremula), gewöhnlicher Traubenkirsche (Prunus padus), Mehlbeere/Vogelbeere (Sorbus) und Birke (Betula), die sich mit den Sträuchern abwechseln. Außerdem gibt es viele Lichtungen mit gemischter Gras-Steppe.

IV. die Wald- bzw. Wiesen-Zone befindet sich in den Bergen auf einer Höhe von $\mathrm{r} 800-2000 \mathrm{~m}$ bis $3000 \mathrm{~m}$ ü. NN. Diese Zone besteht aus einem Mischwald-Gürtel und einem Nadelwald-Gürtel. Als Bodenarten lassen sich hier grauer Gebirgswaldboden und Tschernosem der Gebirgswiese nachweisen. Der Sommer ist kurz und weist gemäßigte Temperaturen auf. Häufig regnen die Wolken sich hier ab, wodurch der jährliche Niederschlag sehr hoch sein kann. Durch eine geschlossene Schneedecke von Oktober bis April ist Ackerbau nicht möglich. Die drei bis vier Sommermonate lassen lediglich die Sommerweide zu.

V. die Hochgebirgszone besteht aus einem alpinen und einem Gletscher-Schnee-Gürtel. Sie befindet sich auf Höhen über $3000 \mathrm{~m}$ ü. NN. Die Winterperiode ist hier lang und kalt, die Sommer sind kurz und kühl. Im niedriger gelegenen alpinen Gürtel dominieren Hochgebirgswiesen, die für eineinhalb bis zwei Monate im Jahr als Sommerweiden für Schafe und Pferde genutzt werden können.

Bei 3500 m ü. NN liegt die Gletscher-Grenze. Darüber befinden sich nur Gletscher, Firnfelder und Felsen.

Das Arbeitsgebiet der vorliegenden Studie umfasst das südöstliche Siebenstromland in Kasachstan (Karte 3). Die nördliche Begrenzung bilden der Kapčagaj-Stausee und der Ili-Fluss. Die Südgrenze ver- 
läuft vom Transili-Alatau über die Nordabhänge des Kungej-Alatau im Westen bis zum hochgelegenen Plateau Kegen im Osten. Die westliche Grenze bilden die Flüsse Große Almatinka und Kleine Almatinka in der Nähe von Almaty, während im Osten die Grenze des Arbeitsgebietes entlang der Ausläufer des Transili-Alatau verläuft.

Dieser Raum umfasst vier unterschiedliche Landschaftseinheiten (Karte 4) und drei Klimazonen (Karte 5).

Auf einer relativ kurzen Strecke, die lediglich $27 \mathrm{~km}$ Luftlinie von den Hochgebirgen des nördlichen Tien-Shans bis zur nördlich anschließenden Ebene misst, sind sehr große Höhenunterschiede festzustellen ${ }^{62}$. Dem Hochgebirge des Tien-Shans ist im Norden ein Lösshügelland vorgelagert (Karte 4). Darauf folgen ausgedehnte Schwemmfächer mit anschließenden Schwemmlandebenen (Gass 20I1a, 58, Fig. I; Blättermann 20I3, Kap. 4.2.). Hoch in den Gebirgen und Bergtälern herrschen kühle und feuchte Bedingungen des nivalen Klimas mit relativ kurzen, aber niederschlagsreichen Sommern (Karte 5). Der Niederschlag fällt zumeist als Schnee (Fizičeskaja geografija Respubliki Kazachstan I998, I27-I28). Die Landstriche unterhalb der Gebirgszone sind dagegen durch ein gemäßigtes Klima geprägt. Die Niederschlagsmengen nehmen ab, was in den Sommermonaten zu Dürreperioden führen kann. Dafür sind jedoch die Wintermonate milder. Die Steppe, die weiter nördlich liegt, weist ein stark kontinentales Klima auf. Die fast fehlende Wolkendecke führt zu starken Schwankungen zwischen Tagund Nachttemperaturen. Der geringe Niederschlag und die niedrige Luftfeuchtigkeit sind weitere Charakteristika dieses Klimas (Fizičeskaja geografija Respubliki Kazachstan I998, 30-74, I93).

Die unterschiedlichen klimatischen Bedingungen führen in einem solch relativ kleinen Raum zur Entstehung verschiedener Vegetationszonen, die in kombinierter Nutzung vorteilhaft für die Viehwirtschaft sein können (Gass 20ıIa, 58, Fig. 2; Gass 20ırb, 210). In Anlehnung an die „Lebensräume“ nach Abolin (siehe oben) können in den Wintermonaten die Zone der Artemisia-Steppe und im Sommer die Zone der gemischten Grassteppe, der Wald und die Wiesen als Weidegebiete für die unterschiedlichen Tierarten der Nomaden genutzt werden. Derart wird im südöstlichen Siebenstromland eine ganzjährige Viehweide mit zyklischer Nutzung unterschiedlicher Weidegebiete möglich.

Da die Vorgebirge durch ihre milden Winter und die Nutzung von Irrigationssystemen in den Sommermonaten hervorragende Anbaugebiete sind, stellt sich das Siebenstromland insgesamt als attraktiver Raum für Ackerbau und Weidewirtschaft dar (Gass 20Iıa, 58; Gass 20Iıb, 2I0).

Es erscheint letztlich vorstellbar, dass das Siebenstromland bereits in der Vorgeschichte Überlappungsbereich unterschiedlicher Wirtschaftsformen war und sowohl von sesshafter als auch von nomadisierender Bevölkerung besiedelt und wirtschaftlich genutzt wurde.

62 Pik Talgar - 4973 (4975) m; das nördlich vom TransiliAlatau gelegene Flachland, die Südgrenze - $973 \mathrm{~m}$ ü.
NN (Zailijskij Alatau 1972; Google Earth, Google TM 2010 [10. 06. 2010]). 